\title{
Impacto de un programa de optimización de uso de antimicrobianos en un hospital pediátrico de tercer nivel en Panamá
}

\author{
Impact of an antimicrobial stewardship program in a pediatric third level \\ hospital in Panama
}

Magda Rojas-Bonilla', Kathia Luciani', Denis Jara² e Israel Ríos-Castillo ${ }^{3}$

'Departamento de Infectología Pediátrica. Hospital de Especialidades Pediátricas Omar Torrijos Herrera. Panamá, Ciudad de Panamá.
Unidad de Farmacia. Hospital de Especialidades Pediátricas Omar Torrijos Herrera. Panamá, Ciudad de Panamá.
ªcultad de Medicina, Universidad de Panamá.
Financiamiento:
Los autores declaran la ausencia de conflictos de interés.

Recibido: 4 de diciembre de 2018 / Aceptado: 22 de noviembre de 2019

\section{Resumen}

Introducción: Los programas de optimización de uso de antimicrobianos (PROA) se enfocan en el uso apropiado de antimicrobianos para ofrecer mejores resultados clínicos y menores riesgos de eventos adversos. Objetivos: Comparar consumo y costos de antimicrobianos antes y después de instauración de un programa de regulación de antimicrobianos y describir la proporción de resistencia de bacterias prioritarias. Métodos: Estudio cuasi-experimental, retrospectivo y prospectivo, descriptivo y analítico, que comparó el consumo y costo de antimicrobianos en un período pre- intervención (2007-2010) y un período post-intervención (2011-2017). Se realizó análisis descriptivo de resistencias bacterianas prioritarias. Resultados: El consumo de gentamicina, vancomicina, meropenem, cefotaxima, ceftazidima e imipenem disminuyó significativamente en el período postintervención comparado con el período pre-intervención $(p<0,05)$, mientras que el consumo de amikacina, piperacilina/tazobactam, cefepime y levofloxacina en el período post-intervención mostró un aumento significativo. La reducción de costos no fue significativa para gentamicina, vancomicina, meropenem, cefotaxima, ceftazidima e imipenem. Para amikacina, cefepime, piperacilina/tazobactam y levofloxacina el aumento de costos no fue significativo. Los aislamientos de Acinetobacter baumannii, Klebsiella pneumoniae, Staphylococcus aureus y Enterococcus faecalis disminuyeron durante el período post-intervención. Conclusión: el PROA demostró disminución en consumo y costos de algunos antimicrobianos.

Palabras clave: Antimicrobianos; hospital pediátrico; bacteria.

\begin{abstract}
Background: Antimicrobial Stewardship Programs (ASP) focus in the appropriate use of antimicrobials to improve clinical results and minimize risk of adverse events. Aims: To compare consumption and costs of antimicrobials before and after the establishment of an antimicrobial stewardship program and to describe the resistance proportion of priority bacteria. Methods: Quasi-experimental, retrospective and prospective, descriptive and analytical study, to compare consumption and costs of antimicrobials in a pre- intervention period (2007-2010) and a post- intervention period (20112017). Additionally, a descriptive analysis of bacterial resistance from 2010 was performed. Results: Gentamicin, vancomycin, meropenem, cefotaxime, ceftazidime and imipenem consumption decreased significantly in the post-intervention period compared to the pre-intervention period $(\mathrm{p}<0.05)$ while consumption of amikacin, piperacillin/tazobactam, cefepime and levofloxacin increased significantly in the post-intervention period. The reduction in costs was not significant for gentamicin, vancomycin, meropenem, cefotaxime, ceftazidime and imipenem, meanwhile, costs increased for amikacin, piperacillin/tazobactam, cefepime and levofloxacin, but this was not significant. The isolation of Acinetobacter baumannii, Klebsiella pneumoniae, Staphylococcus aureus and Enterococcus faecalis decreased during the post-intervention period. Conclusion: The ASP showed a decrease in consumption and costs of some antimicrobials.
\end{abstract}

Keywords: Antibiotics; pediatric hospital; bacteria.

\footnotetext{
Correspondencia a:

Magda Rojas-Bonilla

magda3377@gmail.com
} 


\section{Introducción}

L a resistencia bacteriana es un problema de salud pública que ha escalado en los últimos años y actualmente se identifica como una crisis global. Esta situación es debida, en parte, al uso indiscriminado de los antimicrobianos, combinado con la falta de descubrimiento de nuevas moléculas y desarrollo de éstas ${ }^{1}$. En el año 2014, la Asamblea Mundial de la Salud de OMS y los mandatarios de los países desarrollados declararon y precisaron la búsqueda de soluciones urgentes para la crisis emergente, resultando en "un plan de acción global contra la resistencia a los antimicrobianos" ${ }^{2}$.

Los programas de optimización de uso de antimicrobianos (PROA) han sido definidos como una intervención coordinada, designada para mejorar y medir el uso apropiado de agentes antimicrobianos mediante la promoción de la selección del régimen antimicrobiano óptimo, incluyendo la dosis, duración de la terapia y la ruta de administración" ${ }^{\prime 3,4}$.

Algunos de los beneficios significativos demostrados de los PROA incluyen: i) reducción en $20-50 \%$ en el uso de antimicrobianos; ii) reducción significativa en los costos, variable de acuerdo con el país y programa; iii) minimización de los efectos adversos a los fármacos; iv) reducción de infecciones por Clostridium difficile; v) reducción de las infecciones asociadas al cuidado de la salud debido a la reducción en el tiempo de hospitalización; vi) minimización de interacciones medicamentosas; y vii) disminución de la resistencia microbiana ${ }^{5}$.

En la población pediátrica hay un gran número de prescripción de antimicrobianos. El proceso de las enfermedades, los patrones de resistencia, estrategias de dosificación y los antimicrobianos más comúnmente prescritos varían en comparación con los adultos. Por lo tanto, es importante implementar el PROA ${ }^{6,7}$ identificando objetivos específicos y áreas de prioridades en la población pediátrica, las que varían de acuerdo con la epidemiología local ${ }^{6}$.

Por otra parte, la evidencia de los PROA en pediatría es limitada. El impacto de estos programas y la decisión del mejor enfoque, así como las métricas preferidas para su evaluación, no han sido completamente definidos ${ }^{7}$. Otros centros que han abordado PROA en Pediatría reportan que donde existía vigilancia de antimicrobianos por parte de un infectólogo, como en nuestro caso, los antimicrobianos restringidos disminuyeron de 1.250 a 988 dosis por cada 1.000 pacientes/días, y los antimicrobianos no restringidos de 1.839 a 916 dosis por cada 1.000 pacientes/días ${ }^{8}$. Un estudio en hospitales de Estados Unidos de América (E.U.A.) reportó una disminución en el uso de todos los antimicrobianos de 883 días de terapia (DDT) a 787 DDT por cada 1.000 días $^{9}$. Independientemente de las métricas utilizadas en el análisis del PROA, los antimicrobianos restringidos y los no restringidos mostraron reducción en su uso? ${ }^{7}$.

En Panamá se demostró la disminución en el uso de antimicrobianos con base en resultados de DDT con diferencias significativas, con posterioridad a la implementación de PROA en sala de neonatología ${ }^{10}$.

Respecto a costos en estudios PROA en Pediatría, un hospital pediátrico en Filadelfia, E.U.A., mostró costos ahorrados por el orden de US\$50,090 en un período de cuatro meses luego de instaurar un PROA con la supervisión de infectología ${ }^{11}$. Un análisis longitudinal de ahorro sostenido en un hospital de Baltimore, E.U.A. ${ }^{12}$ reportó costos ahorrados por el orden de US\$103,787 por año. Asimismo, dos revisiones sistemáticas ${ }^{7,13}$ acerca de PROA en pediatría demostraron que, independientemente de las estrategias utilizadas, estos programas reducen la utilización de antimicrobianos en costos y en errores de prescripción, sin aparente impacto negativo en la seguridad del paciente.

En Panamá, el sistema de salud está a cargo de dos entidades financiadoras de la salud pública y la atención médica: la Caja de Seguro Social (CSS) y el Ministerio de Salud (MINSA), ente rector del Sistema Nacional de Salud $^{14}$. Estas dos entidades aportan cerca de $70 \%$ de los recursos utilizados, dejando $30 \%$ al gasto de bolsillo de los ciudadanos.

La CSS, al que pertenece el Hospital de Especialidades Pediátricas Omar Torrijos Herrera (HEPOTH), dirige programas de salud principalmente de tipo curativo y destinado a proteger la salud de la población asegurada. La cobertura de salud de la CSS es de $60 \%$ de la población y el HEPOTH atiende a 5\% de los niños bajo 15 años de edad asegurados, referidos de todas partes del país ${ }^{14}$.

En el país no existe una normativa nacional para el uso apropiado de antimicrobianos. Basado en la creciente problemática de la resistencia bacteriana intrahospitalaria reportada, el HEPOTH de la CSS decidió instaurar la normativa para el uso racional de antimicrobianos. El HEPOTH es un hospital pediátrico con 123 camas en sala, 22 camas en cuartos de urgencias y 6 camas en unidad de cuidados intensivos pediátricos y 6 camas en unidad de semi-intensivos. Cuenta con 26 especialidades y 125 especialistas. En 2016, registró 3.430 admisiones y 1.519 egresos semestrales, con un porcentaje de ocupación semestral de 73,2\% para el mismo año. El presupuesto anual destinado a compra de medicamentos en 2017 fue de aproximadamente 3 millones de dólares americanos. En este contexto, los objetivos del presente estudio son: i) comparar el consumo y costos de los antimicrobianos antes y después de instauración de un programa de regulación de antimicrobianos; y ii) describir la proporción de resistencia de bacterias prioritarias. 
Distribución de medicamentos de la CSS. Posteriormente, se calculó el consumo de viales por mes y por año.

\section{Tipo y diseño de estudio}

Se realizó un estudio con diseño cuasi-experimental, retrospectivo y prospectivo, descriptivo y analítico. Las variables evaluadas fueron el uso de antimicrobianos, consumo y costos, comparándolas en el período preintervención (enero 2007 a diciembre 2010) y período post intervención (enero 2011 a diciembre 2017). Adicionalmente, se evaluó la proporción de resistencia bacteriana durante el período post-intervención, a partir del año 2010.

\section{Diseño de intervención}

Se aplicaron medidas restrictivas que consistían en una lista de los antimicrobianos regulados de aprobación por el médico infectólogo para las diferentes salas. En horas donde no había presencia de infectólogo, la primera dosis se entregó y en el turno siguiente, si había disponibilidad del especialista, la dosis se entregó a través de una interconsulta bajo aprobación del infectólogo. El departamento de farmacia dispensó los antimicrobianos (regulados o no) por un período máximo de 10 días; si el médico tratante consideró necesario prolongar la duración de la terapia, solicitó una re-evaluación por infectología.

Los antimicrobianos regulados fueron cefalosporinas de $2^{\circ}, 3^{\circ}$ y $4^{\circ}$ generación, piperacilina/tazobactam, vancomicina, teicoplanina, oxazolidonas, tetraciclinas, cotrimoxazol endovenoso, polimixina $\mathrm{B}$, aminoglucósidos, quinolonas, metronidazol endovenoso y carbapenems.

Desde el inicio del PROA se realizó rotación de gentamicina por amikacina. Desde entonces se utiliza de primera línea cefepime para las neutropenias febriles en lugar de ceftazidima, y piperacilina/tazobactam en lugar de meropenem para las neumonías asociadas a la atención de salud.

El equipo de PROA que implementó la intervención está formado por dos infectólogos pediatras de tiempo completo y tres miembros del equipo de farmacia que rotan por las diferentes salas de hospitalización. El PROA del HEPOTH se inició a partir del año 2010 mediante la confección de normas de regulación de antimicrobianos aprobadas por el director médico local y el jefe de farmacia.

\section{Cuestionario de recolección de datos}

La recolección de datos respecto al consumo de viales de antimicrobianos (viales/año) se realizó revisando el registro del almacén de medicamentos (evaluando las entradas de medicamentos y salidas dispensadas a las salas), en los períodos 2007 a 2010 y 2011 a 2017. Con respecto a los costos, éstos se obtuvieron mediante la revisión del listado de medicamentos con el precio unitario. Los costos anuales fueron proporcionados por el Centro de

\section{Susceptibilidad de microorganismos aislados}

La susceptibilidad de los microorganismos aislados de muestras clínicas significativas fue obtenida de hemocultivos, urocultivos, secreciones y líquido cefalorraquídeo, descartándose las colonizaciones. La susceptibilidad se determinó utilizando el equipo automatizado Vitek- $2^{\circledR}$ Compact (VITEK 2; BioMérieux, Inc., Hazelwood, MO), siguiendo los puntos de corte propuesto por el Instituto de Estándares Clínicos y de Laboratorio (CLSI, por sus siglas en inglés) ${ }^{15}$. Se presentan los resultados sólo de las bacterias que, según la OMS, son de crítica y de alta prioridad $^{16}$.

\section{Análisis estadísticos de datos}

Todos los análisis se realizaron con el programa Stata 11.0 (StataCorp, College Station, Texas, USA). Para verificar la distribución de los datos se empleó la prueba de normalidad Shapiro Wilks. Debido a que el consumo y costo de antimicrobianos seguía una distribución no asimétrica, se empleó estadística no paramétrica para presentar los datos. Las variables continuas se presentan como mediana y rango intercuartílico (RIQ) (p75-p25). Para comparar el consumo y costo de cada antimicrobiano antes y después de la implementación del PROA, se dividió el consumo pre-intervención y post-intervención y se aplicó para cada período la prueba estadística no paramétrica prueba de los rangos con signo de Wilcoxon para comparación de medianas. El valor de la significancia se estableció cuando el valor $\mathrm{p}$ asociado a las pruebas fue menor de $5 \%(p<0,05)$.

\section{Resultados}

En total se evaluó el consumo y costo para 10 antimicrobianos restringidos y de mayor utilización en la institución durante los períodos 2007 a 2010 y 2011 a 2017.

\section{Análisis de comparación de consumo (viales por año)}

La Tabla 1 muestra el consumo de los antimicrobianos, las medianas y RIQ del período pre-intervención y postintervención. El consumo de gentamicina, vancomicina, meropenem, cefotaxima y ceftazidima disminuyó en el período-post-intervención comparado con el período pre-intervención de 401,5 (149) a $98(152,5)$ para gentamicina; de 281 (134) a 172,5 (128) viales para vancomicina; de $265(205)$ a $246(173,5)$ para meropenem; de $1.212,5(592,5)$ a $1.019(300,5)$ viales para cefotaxima; y de 425,5 (202,5) a $150(152,5)$ viales para ceftazidima. Con relación al consumo de imipenem, en el período 


\begin{tabular}{|c|c|c|c|c|}
\hline Antimicrobiano & $\begin{array}{c}\text { Período pre-intervención } \\
\text { Mediana (RIQ) }\end{array}$ & $\begin{array}{c}\text { Período post-intervención } \\
\text { Mediana (RIQ) }\end{array}$ & z & $\mathbf{p}^{*}$ \\
\hline Gentamicina & $401,5 \quad(149)$ & $98 \quad(152,5)$ & 6,031 & 0,000 \\
\hline Amikacina & $122,5 \quad(81)$ & $294,5(141,5)$ & $-5,590$ & 0,000 \\
\hline Vancomicina & $281 \quad(134)$ & $172,5 \quad(128)$ & 5,528 & 0,000 \\
\hline Meropenem & $265 \quad(205)$ & $246 \quad(173,5)$ & 2,205 & 0,027 \\
\hline Cefotaxima & $1.212,5(592,5)$ & $1.019 \quad(300,5)$ & 3,826 & 0,000 \\
\hline Ceftazidima & $425,5(202,5)$ & $150 \quad(152,5)$ & 5,841 & 0,000 \\
\hline Cefepime & (13) & $209 \quad(232)$ & $-5,764$ & 0,000 \\
\hline Imipenem & $31,5 \quad(71,5)$ & (0) & 5,150 & 0,000 \\
\hline Piperacilina/tazobactam & $12,5 \quad(26,5)$ & $54 \quad(52,5)$ & $-4,124$ & 0,000 \\
\hline Levofloxacina & (9) & $8,5 \quad(21)$ & $-2,114$ & 0,034 \\
\hline
\end{tabular}

pre-intervención fue de $31,5(71,5)$ y para el período post-intervención no se registró consumo. Al evaluar la mediana de consumo por período, todos mostraron diferencia estadísticamente significativa (prueba de los rangos con signo de Wilcoxon, $\mathrm{p}<0,05$ ).

Por su parte, el consumo de amikacina en el período post intervención mostró un aumento en la mediana (RIQ), siendo de 294,5 $(141,5)$ viales con respecto al período pre-intervención de 122,5 (81) viales (prueba de los rangos con signo de Wilcoxon, $\mathrm{p}<0,05)$. A diferencia de otros antimicrobianos, la mediana (RIQ) de consumo de cefepime, luego del PROA (período post-intervención), fue mayor que el período-pre-intervención (prueba de los rangos con signo de Wilcoxon, $\mathrm{p}<0,05$ ). Asimismo, la mediana (RIQ) de consumo de piperacilina/tazobactam se incrementó a $54(52,5)$ viales en el período post-intervención, en comparación con el período pre-intervención de $12,5(26,5)$ unidades. Levofloxacina mostró un consumo mayor luego del PROA con una mediana (RIQ) de 8,5 (21) viales en el período post-intervención en comparación con el período pre-intervención.

El consumo de antimicrobianos en dólares mostró una reducción de \$92,843.02 en el período post-intervención.

\section{Análisis de comparación de costos}

No se observaron diferencias significativas con relación a los costos reales durante la implementación del PROA. Los antimicrobianos que mostraron reducción de costo del período pre-intervención al post-ntervención, fueron gentamicina, de US\$ 1400,41 $(1438,33)$ a US\$ $281,90(274,96)$; vancomicina, de US\$ 7220,62 (1430,84) a US\$ 3684,24 (3397,46); meropenem, de US\$ 71958,90 $(19830,60)$ a US\$ 24222,50 (13005,80); cefotaxima, de US\$ 19684,50 $(28114,58)$ a US\$ 4913,64 (1493,52); ceftazidima, de US\$ 6738,88 $(2452,89)$ a US\$ 1303,56 (1140,32); e imipenem, de US\$ 6241,93 $(6160,93)$ a $326,16(2376,40)$. Por su parte, los antimicrobianos que incrementaron el costo fueron amikacina, de US\$ 741,71 $(283,18)$ a US\$ $1725,75(1544,43)$; cefepime, de US\$ $758,10(579,90)$ a US\$ 9061,20 $(2428,80)$; y, piperacilina/ tazobactam, de US\$ 2538,03 $(1685,32)$ a US\$ 2955,37 $(633,32)$. Los costos para levofloxacina aumentaron igualmente (Tabla 2).

\section{Resistencia bacteriana}

La Figura 1 muestra el número de casos de pacientes infectados con las bacterias prioritarias entre los años 2010 y 2016. En el año 2010, 14,5\% de los casos infecciosos correspondió a Klebsiella pneumoniae y 5,6\% a Acinetobacter baumannii. En 2016 se encontró 19,6 y 19,7\% para Enterobacter cloacae y Pseudomonas aeruginosa, respectivamente, y $11,0 \%$ de $K$. pneumoniae.

La Figura 2 presenta la proporción (\%) de A. baumannii según la resistencia a distintos antimicrobianos. Se observa mayor resistencia en los años 2010 y 2011 para amikacina cefepime, gentamicina y meropenem. Entre los años 2012 y 2015, disminuyó para todos los antimicrobianos, siendo está por debajo de $20 \%$. Sin embargo, aumentó en 2016 para cefepime (25\%).

La Figura 2 muestra que la resistencia bacteriana de E. coli para amikacina y meropenem fue uniforme en el período 2010 y 2016. Se observa un aumento de la misma 


\begin{tabular}{|c|c|c|c|c|}
\hline Antimicrobiano & $\begin{array}{l}\text { Período pre-intervención } \\
\text { Mediana (RIQ) }\end{array}$ & $\begin{array}{l}\text { Período post-intervención } \\
\text { Mediana (RIQ) }\end{array}$ & z & $p^{*}$ \\
\hline Gentamicina & $1400,41(1438,33)$ & $281,90(274,96)$ & 1,826 & 0,067 \\
\hline Amikacina & $741,71(283,18)$ & $1725,75(1544,43)$ & $-1,461$ & 0,144 \\
\hline Vancomicina & $7220,62(1430,84)$ & $3684,24(3397,46)$ & 1,826 & 0,067 \\
\hline Meropenem & $71958,90(19830,60)$ & $24222,50(13005,80)$ & 1,826 & 0,067 \\
\hline Cefotaxima & $19684,5028114,58)$ & $4913,64(1493,52)$ & 1,826 & 0,067 \\
\hline Ceftazidima & $6738,88(2452,89)$ & $1303,56(1140,32)$ & 1,826 & 0,067 \\
\hline Cefepime & $758,10(579,90)$ & $9061,20(2428,80)$ & $-1,826$ & 0,067 \\
\hline Imipenem & $6241,93(6160,93)$ & $326,16(2376,40)$ & 1,826 & 0,067 \\
\hline Piperacilina/tazobactam & $2538,03(1685,32)$ & $2955,37(633,32)$ & $-0,730$ & 0,465 \\
\hline
\end{tabular}

en cefepime, sin descenso desde el año 2011 hasta el año 2016. Gentamicina, por otro lado, disminuyó en el año 2014 y 2015 con poca elevación en el año 2016, siendo su punto más alto el año 2013.

La Figura 3 muestra la tendencia de la resistencia en $K$. pneumoniae. No se encontró un porcentaje mayor a 5\% en el período 2010-2016 para meropenem y amikacina. Se observó una elevación en los años 2012 para cefepime y gentamicina entre 30 y $35 \%$, con un descenso hasta el año 2015 para ambos antimicrobianos. En el año 2016 cefepime presentó un aumento por sobre $25 \%$ y gentamicina disminuyó por debajo de 5\%. La Figura 3 también presenta la resistencia bacteriana de $P$. aeruginosa por año a distintos antimicrobianos. Amikacina presentó un aumento de $20 \%$ en el año 2012 hasta casi $30 \%$ en el año 2015, pero en el año 2016 disminuyó a 20\%. La respuesta a cefepime, gentamicina y meropenem fue similar entre los años 2010 y 2013. Luego de este período se presentó un aumento entre 10 y $20 \%$, y la misma disminuyó para los tres antimicrobianos en el año 2016, aproximándose a $10 \%$.

La Figura 4 presenta la resistencia de Staphylococcus aureus por año a diferentes antimicrobianos. En el año 2010 , la resistencia a oxacilina fue de $12 \%$ y a clindamicina de 9\%, fluctuando en los años subsiguientes. En 2016, la resistencia a clindamicina descendió a 6\%. Oxacilina mostró su pico más alto en 2014, con $22 \%$ de resistencia y en el 2016 tuvo un descenso a 15\%. En 2010 , la resistencia a levofloxacina era de $0 \%$ y en el año 2016 se reportó en 4\%. No se reportó en el caso de linezolid y vancomicina.

La Figura 4 muestra, además, la resistencia para
Enterococcus faecalis. El año 2010 muestra los picos más altos en ampicilina y levofloxacina con 35 y $25 \%$ de resistencia, respectivamente, las que disminuyeron, y para el año 2016 ya no presentaban resistencia, al igual que vancomicina y linezolid.

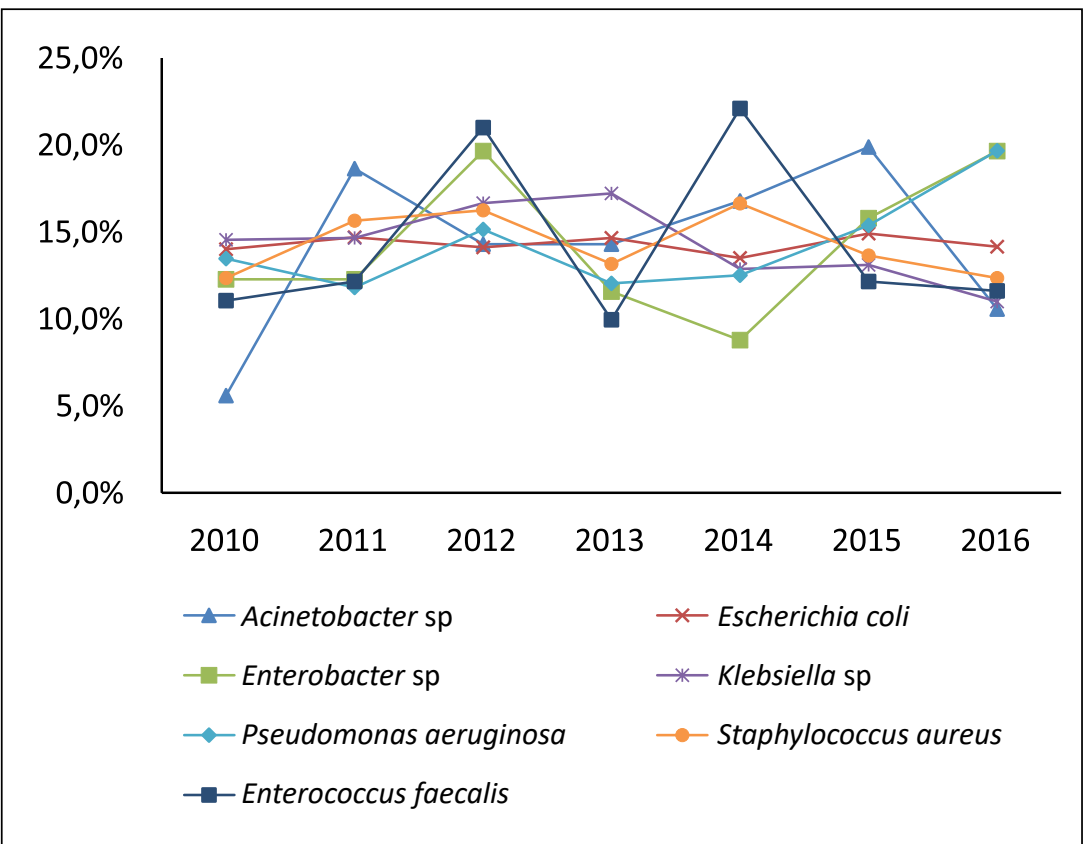

Figura 1. Evolución de casos de pacientes infectados con las bacterias prioritarias desde 2010 a 2016 del Hospital de Especialidades Pediátricas, Panamá. Serie cronológica de casos infectados con bacterias prioritarias. Los marcadores representan la proporción de casos. Fuente: Departamento de Microbiología Hospital de Especialidades Pediátricas. 


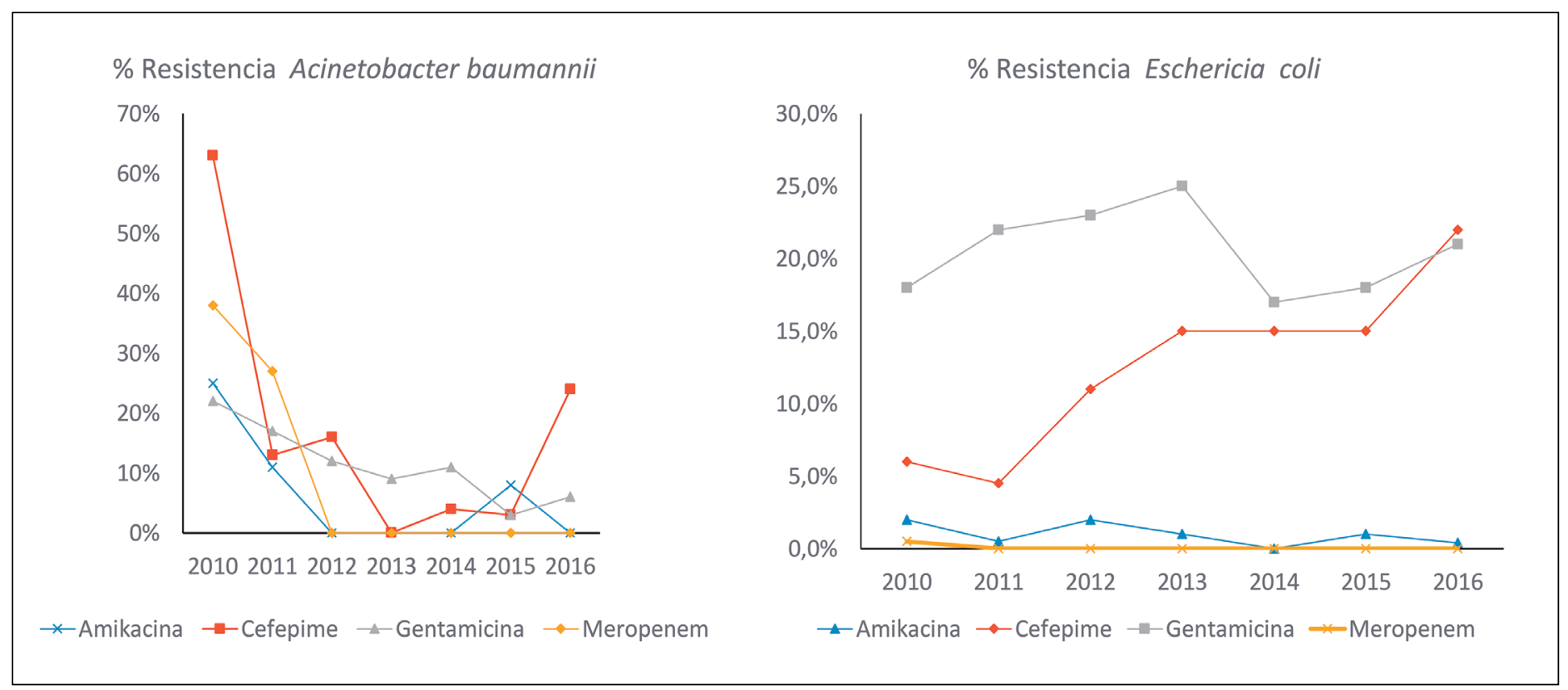

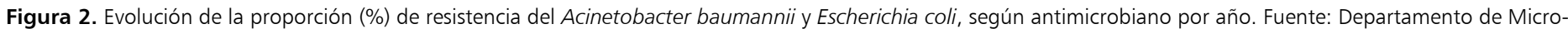
biología del Hospital Especialidades Pediátricas año 2010 a 2016.

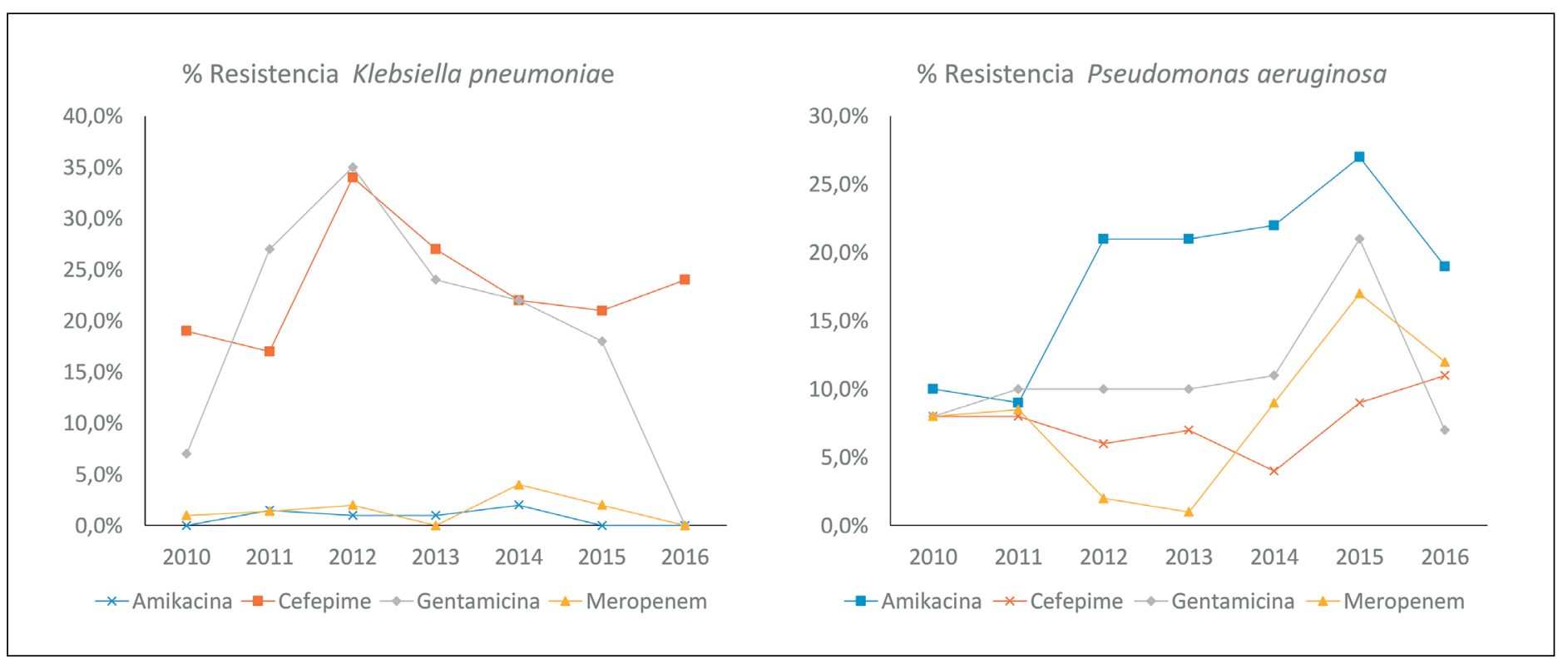

Figura 3. Evolución de la proporción (\%) de resistencia de Klebsiella pneumoniae y Pseudomonas aeruginosa, según antimicrobiano, por año. Fuente: Departamento de Microbiología del Hospital de Especialidades Pediátricas año 2010 a 2016.

\section{Discusión}

Este es el primer PROA realizado en Panamá que entrega datos sobre el consumo y costo de los antimicrobianos, antes y después de la instauración del programa, así como de la proporción de resistencias bacterianas prioritarias. De acuerdo con nuestros resultados, el PROA fue efectivo para disminuir consumo y costos de algunos de los antimicrobianos evaluados. Villegas y cols., describen que el objetivo de las políticas de restricción de antimicrobianos deberá impactar en el costo y consumo, por la limitación en el uso de antimi- 


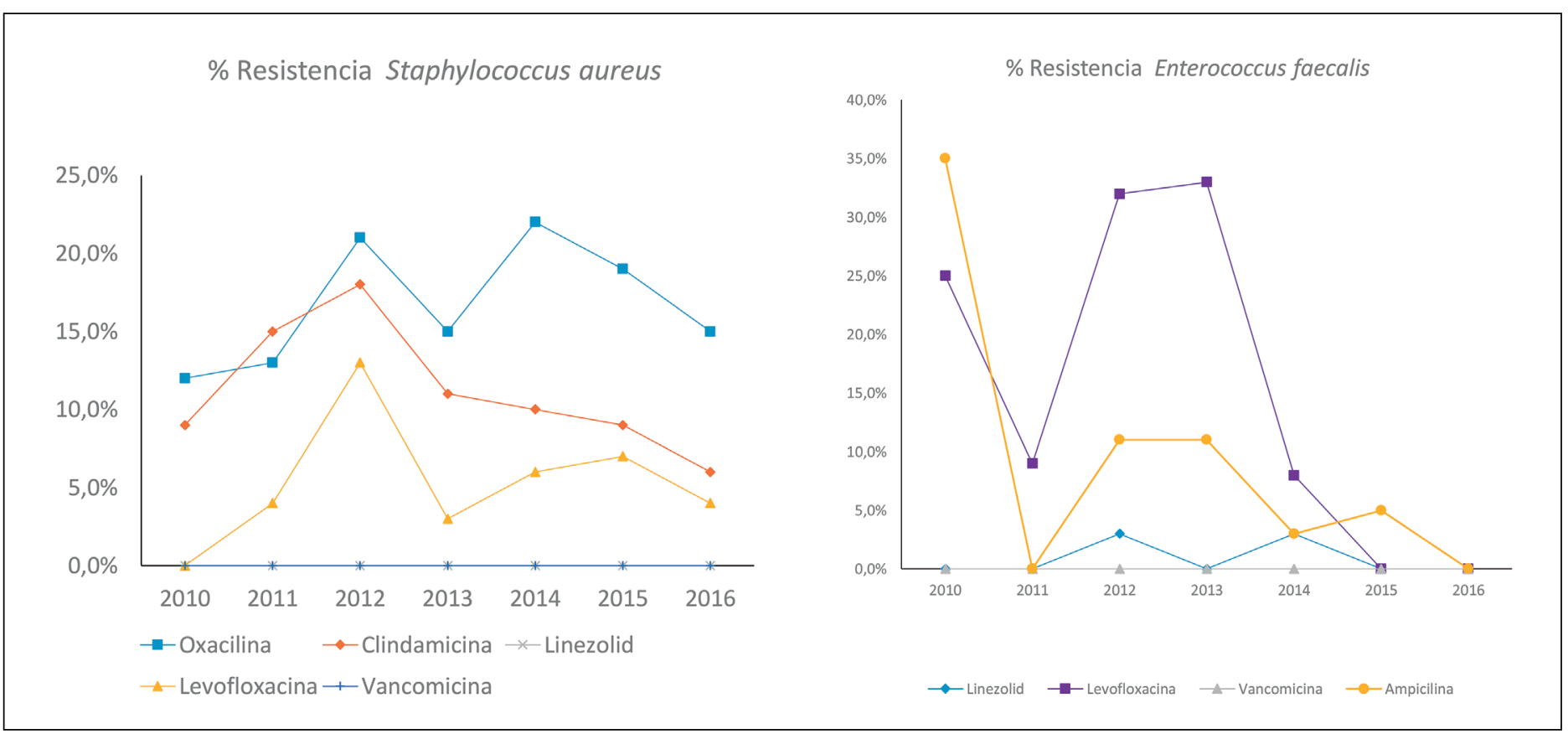

Figura 4. Evolución de la proporción (\%) de resistencia del Staphylococcus aureus y Enterococcus faecalis según antimicrobiano por año. Fuente: Departamento de Microbiología del Hospital de Especialidades Pediátricas año 2010 a 2016.

crobianos con precios más altos o por la reducción en la resistencia bacteriana (a través de la limitación de antimicrobianos con mayor posibilidad de desarrollar presión selectiva) $)^{5}$.

En el HEPOTH hay variables que a través de los años no han aumentado: cantidad de camas disponibles, brotes de enfermedades naturales o de bacterias multi-resistentes y la apertura de nuevos servicios, razón por la cual el consumo mantiene un comportamiento constante.

Con respecto a las variables de costos, los proveedores han variado en ocasiones y los precios han podido fluctuar, pero las políticas de compras se han mantenido en el tiempo sin cambios. Incluso, el desabastecimiento de medicamentos ha suscitado que ciertos medicamentos de amplio espectro hayan aumentado en consumo.

De acuerdo con los resultados obtenidos, si se compara el consumo de antimicrobianos del período-preintervención con el período post-intervención, se observa con respecto al consumo de vancomicina una disminución estadísticamente significativa luego de la instauración del PROA. Esta reducción es consistente con las recomendaciones de las guías internacionales del uso prudente de vancomicina para disminuir infecciones y colonizaciones con enterococo resistente a vancomicina, y emergencia de $S$. aureus resistente a vancomicina (sigla en inglés: VRSA) y/o S. epidermidis resistente a vancomicina (sigla en inglés: VRSE) $)^{17}$.
Desde el inicio del PROA, se realizó la rotación de gentamicina por amikacina. La rotación cíclica de antimicrobianos consiste en restringir de forma determinada y establecida un antimicrobiano durante un determinado período de tiempo, para reintroducirlo posteriormente. De este modo, se pretende evitar la aparición de virulencia bacteriana derivadas de un uso continuado del mismo ${ }^{18}$.

En el presente estudio se observó una disminución significativa de consumo de gentamicina así como una disminución de costos no significativa, además de un aumento en el consumo de amikacina. En Croacia ${ }^{19}$ reportaron que el uso exclusivo de amikacina (frente a gentamicina) redujo significativamente la resistencia de las cepas de bacilos gramnegativos, el número de bacteriemias nosocomiales atribuidas a bacilos gramnegativos y el costo total del uso de antimicrobianos en la UCI.

Con respecto a meropenem, el consumo se redujo significativamente. Similar situación se observó para los costos, aunque ésta no fue significativa. Es importante recalcar que hubo períodos entre 2015 a 2017 de desabastecimiento de otras moléculas que obligaron el uso de meropenem como primera opción para pacientes con diagnóstico de neutropenia febril. Imipenem mostró una disminución en el consumo en el período postintervención significativa y en los costos, aunque de forma no significativa. Esto se puede explicar debido a que es un medicamento de poca prescripción en el hospital. 
Las cefalosporinas de tercera generación mostraron una disminución significativa solamente en el consumo y una disminución de costos no significativa, al instaurarse la restricción de antimicrobianos. Esta medida respondió a las recomendaciones internacionales de restricción de su uso debido a la capacidad de presión selectiva que se crea mediante el uso indiscriminado, generando una plétora de microorganismos con múltiples resistencias. Un estudio realizado en España ${ }^{20}$ en una UCI, reportó que las cefalosporinas fueron los antimicrobianos con mayor impacto en su disminución tras la implantación del PROA.

Era esperado que en el período post-intervención hubiera un aumento en el consumo de ciertas moléculas, como el caso de cefepime y piperacilina/tazobactam, que tuvieron incrementos estadísticamente significativos debido a prescripción del equipo de infectología, ya que cefepime es la primera opción en pacientes con neutropenia y fiebre. Con respecto a piperacilina/tazobactam, este antimicrobiano es la primera opción, en nuestra institución, para casos de neumonía nosocomial e infecciones intra-abdominales complicadas en pacientes hemodinámicamente estables. El aumento de estas dos moléculas también fue descrito en Colombia en un hospital de tercer nivel donde se estudió el impacto del PROA ${ }^{21}$.

Una revisión sistemática con respecto al uso de antimicrobianos en PROA describió que, aunque el consumo total de antimicrobianos disminuía, el consumo de ciertos antimicrobianos podía aumentar. Esto era evidente en varios estudios que mostraban que, a medida que los aminoglucósidos, carbapenems y $\beta$-lactámicos con inhibidores de $\beta$-lactamasas aumentaban, otros antimicrobianos como macrólidos disminuían ${ }^{1}$. Levofloxacina igualmente presentó un aumento estadísticamente significativo con respecto al consumo. El aumento de levofloxacina en el HEPOTH pudo deberse a indicación a pacientes con fibrosis quística por el servicio de neumología.

Con respecto al porcentaje de resistencia bacteriana, se realizó un análisis descriptivo como serie cronológica de pacientes infectados con estas bacterias prioritarias desde el año 2010 hasta el 2016. Los aislamientos de A. baumannii, K. pneumoniae, $S$. aureus y E. faecalis disminuyeron durante el período post-intervención. El aislamiento de $E$. coli se mantuvo constante y fue el microorganismo gramnegativo número uno en el HEPOTH.

Los aislamientos de $P$. aeruginosa y de Enterobacter cloacae aumentaron durante el período post-intervención. El aumento de $P$. aeruginosa y E. cloacae puede deberse a que en los últimos años se ha reportado un incremento en el ingreso de pacientes crónicos con fibrosis quística, neumonías asociadas al ventilador y con mielomeningocele con catéteres vesicales permanentes, respectivamente.

La vigilancia de los aislamientos es importante ya que se sabe que $P$. aeruginosa es un patógeno oportunista frecuente en pacientes con fibrosis quística y que su feno- tipo puede cambiar de una infección aguda a una crónica, además de tener la capacidad de hacer biopelícula, y por ende, ser de difícil erradicación ${ }^{22}$.

Enterobacter cloacae es el aislado más frecuente en muestras humanas del complejo de cepas de Enterobacter. Causante de infecciones en el torrente sanguíneo o infecciones de vías urinarias asociadas a la atención de salud en la última década, es capaz de desarrollar resistencia a través de la sobreproducción de AmpC- $\beta$-lactamasas ${ }^{23}$.

En el HEPOTH, el aislamiento de $S$. aureus resistente a meticilina (SARM) ha disminuido y no se ha tenido aislamiento de $S$. aureus resistente a vancomicina. El porcentaje de resistencia a clindamicina en el HEPOTH, a partir del año 2014 , se mantiene por debajo de $10 \%$ siendo este antimicrobiano una opción para el manejo de infecciones causadas por SARM.

En 2015, el CDC, en su reporte del Programa de Infecciones Emergentes con respecto a SARM, señaló que para ese año las infecciones invasoras asociadas a la atención de salud por SARM habían disminuido en 10\% comparado con el $2014^{24}$. Esto contrasta con un estudio publicado en 2015 en Panamá en adultos, que reportó incidencia de SARM entre 25 y $35 \%$ durante el período del 2007 al $2013^{25}$.

Con respecto a $E$. faecalis, no hay resistencia descrita a vancomicina, mientras que sí ha disminuido en el caso de ampicilina.

La recuperación de la susceptibilidad de los agentes bacterianos a los antimicrobianos se logra como respuesta a una integración de un paquete de medidas en los PROA $\mathrm{y}$ es un resultado que se puede obtener a largo plazo, mientras que los objetivos de las políticas de restricción deben impactar inicialmente en la disminución de costos y consumos ${ }^{5,26}$.

La implementación de un PROA debe asociarse además a un buen diagnóstico de laboratorio y a la implementación de campañas de higiene de manos, limpieza y desinfección de las áreas hospitalarias ${ }^{5}$.

Con respecto a la lista de la OMS de microorganismos de prioridad crítica, no se tuvieron aislados de $\mathrm{A}$. baumannii resistentes a carbapenems, Enterobacteriaceae resistentes a carbapenems, pero sí hubo aislamientos de $P$. aeruginosa resistentes a carbapenems por mecanismos de impermeabilidad, no por producción de carbapenemasas ${ }^{16}$.

Contar con un equipo para el PROA es importante, al igual que acoger e institucionalizar las recomendaciones dadas por el equipo de infectología. Un estudio realizado en el HEPOTH, en Panamá, reportó que las recomendaciones realizadas por infectología fueron seguidas por el médico tratante sólo en $68,7 \%$ de los $\operatorname{casos}^{27}$. En su artículo, Borg y cols., mencionan que el control de la prevención de las infecciones es un microcosmo general de los estándares de calidad y de seguridad dentro de los hospitales y los países. Para mejorar el control de la pre- 
vención de las infecciones se necesitaría, por ende, tomar en cuenta el núcleo de los valores culturales inherentes y subyacentes relevantes para la seguridad y calidad del cuidado del paciente. Las estrategias exitosas del control de infecciones es probable que sean esas que son compatibles con el antecedente cultural donde son implementadas ${ }^{28}$. Por otra parte, hay que impulsar un cambio conceptual en la necesidad de la promoción y apoyo de los PROA.

El estudio tuvo limitaciones. Por una parte, no se utilizó el indicador de días de tratamiento para cuantificar el consumo de medicamentos, ya que, en el año 2010, cuando decidimos iniciar el PROA y medir su impacto, no se contaba con un comité estructurado, no conocíamos las unidades de medida de los indicadores y se decidió expresar e iniciar el registro basado en cantidad de viales de antimicrobianos dispensados. En el caso de los costos, se debió utilizar la variable gasto de antimicrobiano por paciente para así reducir el sesgo de disminución del precio unitario de la molécula. Para solucionar estas limitaciones proponemos un futuro análisis tomando en cuenta variables no analizadas y las unidades de medida.

Además, en el HEPOTH no se cuenta con la capacidad de medir concentraciones plasmáticas de aminoglucósidos ni de vancomicina.

Otra limitación que cabe mencionar es que quienes implementamos el PROA somos quienes realizamos las mediciones de los desenlaces del estudio. Para solucionar este inconveniente se puede sumar al equipo un espe- cialista en estadísticas para el análisis de los resultados.

Una limitación referida al fortalecimiento del PROA en sí, es la necesidad de realizar actividades educativas para los prescriptores y dispensadores de antimicrobianos con adherencia a guías, normas y protocolos de uso de antimicrobianos en las diferentes patologías para así contener la resistencia bacteriana, reportar los resultados a las autoridades para ayudar a regular la prescripción y solicitar a los altos niveles jerárquicos la actualización de las normas clínicas de atención de la institución.

El estudio tiene varias fortalezas. En Panamá no existen normativas nacionales. Este estudio muestra la experiencia de una normativa restrictiva, mediante la implementación del PROA en el HEPOTH constituyéndose en evidencia para el desarrollo de una normativa nacional.

\section{Conclusión}

El PROA del HEPOTH mostró disminución en el consumo y costos de algunos antimicrobianos. Existe la necesidad de contar con un sistema de vigilancia/ registro y reporte que dé cuenta de la implementación, efectividad y eficiencia del PROA a largo plazo, y para ello, es necesario institucionalizarlo como una política de gestión e idealmente aplicarlo a los hospitales de mayor complejidad del país.

\section{Referencias bibliográficas}

1.- Eckart J, Hogan M, Mao Y, Toscani M, Brunetti L. Antimicrobial Stewardship Programs: Effects on clinical and economic outcomes and future directions. J Com Journal. 2017; 24(7): 309318. https://pdfs.semanticscholar.org/c526/666f 6f128c25846d7dd188c88682546ba6c9.pdf.

2.- Organización Mundial de la Salud. Global Action Plan on Antimicrobial Resistance. Ginebra, OMS 2015:1-28 (document WHA68/2015/REC/1, Annex3.) https://apps.who.int/iris/bitstream/hand le/10665/255204/9789243509761-spa.pdf;jsess ionid=EBC2C3E6DC3E9238AAB287EAFBF2 0019? sequence $=1$.

3.- Chung G W, Wu J E, Yeo C L, Chan D, Hsu L Y. Antimicrobial stewardship: A review of prospective audits and feedback systems and an objective evaluation of outcomes. Virulence 2013; 4 (2): 151-7. doi:org/10.4161/viru.21626.

4.- Barlam T, Crosgrove S, Abbo L, MacDougall C, Schuetz A, Septimus J, et al. Implementing an antibiotic stewardship program: Guidelines by Infectious Disease Society of America and the Society for Healthcare Epidemiology of
America. CID 2016 May 62(10): e51- e77. doi:10.1093/cid/ciw118.

5.- Villegas M V, Esparza G, Zurita J. Guía para la Implementación de un Programa de Optimización de Antimicrobianos (PROA) a nivel hospitalario. 1era ed. Asociación Panamericana de Infectología (API); 2016: 1-49. ISBN: 978-9942-14-766-0.

6.- Goldman J, Newland J. New horizons por pediatric antibiotic stewardship. Infect Dis Clin North Am 2015; 29(3): 503-11. doi: 10.1016/j. idc. 2015.05.003.

7.- Smith M, Gerber J, Hersh A. Inpatient antimicrobial stewardship in pediatrics: a systematic review. J Pediatr Infect Dis Soc 2015; 4 (4): e127-e135. doi: 10.1093/jpids/ pin 141.

8.- Di Pentima M C, Chan S, Hossain J. Benefits of a pediatric antimicrobial stewardship program at a Children`s hospital. Pediatrics 2011; 128 (6): 1062-70. doi: 10.1542/peds.2010-3589.

9.- Newland J G, Stach L M, Hedican E B. Impact of a prospective -audits- with-feedback antimicrobial stewardship program at a Children`s hospital. J Pediatr Infect Dis Soc 2012; 1: 179-86. doi: 10.1093/JPIDS/PIS054.
10.- Xamora C, Moreno M T, Norero X. Impacto de políticas de vigilancia en el uso de antibióticos en sala de neonatología del Hospital del Niño, José Renán Esquivel, Panamá julio 2015 a junio 2016. Pediatr Panamá 2018; 47(1): 4-12. http:// docs.bvsalud.org/biblioref/2018/06/885141/ vigilancia-de-antibioticos.pdf.

11.- Metjian T A, Prasad P A, Kogon A, Coffin S, Zaoutis T. Evaluation of an antimicrobial stewardship program at a pediatric teaching hospital. Pediatr Infect Dis J 2008; 27: 106-11. doi: 10.1097/INF.0b013e318158603a.

12.- Sick A C, Lehmann C U. Sustained savings from a longitudinal cost-analysis of an internetbased pre-approval antimicrobial stewardship program. Infect Control Hosp Epidemiol 2013; 34: 573-80. doi: 10.1085/iche.1582.

13.- Magsarili H, Girotto J, Bennett N, Nicolau D. Making the case for Pediatric Stewardship Programs. Pharmacology 2015; 2 (11): 115-25. doi: 10.1002/par.1647.

14.- Mayo M. Análisis de Situación de Salud Panamá 2018. Macro Visión Nacional de Salud. Ministerio de Salud de Panamá. 1-233.

15.- Weinstein M, Patel J, Campeau S, Eliopoulos G, Galas M, Humphries R, et al. CLSI. 
Perfomance Standards for Antimicrobial Susceptibility Testing. 28th ed. CLSI supplement M100. Wayne PA. Clinical and Laboratory Standards Institute, 2018 vol 38(3).

16.- Organización Mundial de la Salud. Antibacterial Agents in Clinical Development. Ginebra, OMS 2017: 1-48. https://apps.who. int/iris/bitstream/handle/10665/258965/WHOEMP-IAU-2017.11-eng.pdf? sequence $=1$.

17.- Stevens D L, Bisno A L, Chambers H F, Dellinger E P, Goldstein E J, Gorbach S L. Practice guidelines for the diagnostics and management of skin and soft tissue infection CID 2014; 59 (2): e10-e52. doi: 10.1093/cid/ ciu296.

18.- Pujol M, Gudiol F. Evidence of antibiotic cycling in control of resistance. Curr Opin Infect Dis 2010; 14: 711-5. PMID 11964889.

19.- Francetic I, Kalenic S, Huic M, Mercep I, Makar-Ausperger K, Likic R, et al. Impact of aminoglycoside cycling in six tertiary intensive care units: prospective longitudinal interventional study. Croat Med J. 2008; 49: 207-14. doi: 10.3325/cmj.2008.2.207.

20.- Ruiz- Ramos J, Gordon Sahuguillo M.
Ramírez Galleymore P, Frasquet J, Broch Porcar MJ, Salavert Lleti M. Implementación de un programa de optimización de antimicrobianos en una unidad de pacientes críticos. Premios Profesor Barea $13^{\mathrm{a}}$ edición. 2015.

21.- Pallares C, Cataño J. Impacto del uso racional de antimicrobianos en una clínica de tercer nivel en Colombia. Rev Chilena Infectol 2017; 34 (3): 205-11. doi.org/10.4067/S071610182017000300001.

22.- Vandeplassche E, Sass A, Lemarcq A. In vitro evolution of Pseudomonas aeruginosa AA2 biofilms in the presence of cystic fibrosis lung microbiome members. Sci Rep. 2019; 9 (1): 128-39. doi:10.1038/S41598019-49371-y

23.- Mezzatesta M L, Gona F, Stefani S. Enterobacter cloacae complex: clinical impact and emerging antibiotic resistance. Future Microbiol. 2012; 7 (7): 887-902. doi:10.2217/ fmb.12.61.

24.- Centers for Disease Control and Prevention. Active Bacterial Core Surveillance Report. Emerging Infectious Program Network MRSA,
2015. Disponible en: https://www.cdc.gov/hai/ eip/pdf/2015-MRSA-Report-P.pdf.

25.- Conte E, Morales Y, Higuera G, Toro J, Moreno J, Herrera V. Estudio sobre resistencia bacteriana y uso racional de antibióticos en Panamá. 2015. Instituto Conmemorativo Gorgas de Estudio de la Salud. Disponible en: https://www.gorgas.gob.pa/SIGMED/Inicio. htm.

26.- Morency-Potvin P, Schwartz D N, Weinstein R A. Antimicrobial stewardship: how the microbiology laboratory can right the ship. Clin Microbiol Rev 2016; 30(1): 381-407. doi: 10.1128/CMR.00066-16.

27.- Cernuda R, Bernardino D, Luciani K. Características de la prescripción de antibióticos en salas de hospitalización el HEPOTH, octubre a diciembre 2016. Pediátr Panamá 2017; 46 (3): 12-21. http://docs.bvsalud.org/ biblioref/2018/02/877517/2017-46-3-12-20.pdf.

28.- Borg M A. Cultural determinants of infection control behaviour: understanding drivers and implementing effective change. J Hosp Infect 2014; 86 (3): 161-8. doi.org/10.1016/j. jhin.2013.12.006. 